hypoxia or ischemia. Diagnosis is confirmed by muscle biopsy and quantitative analysis of respiratory chain enzymes. Many CNS and muscle diseases that are not primary MC may involve mitochondrial alterations; these include Pompe disease, spinal muscular atrophy, infantile spinocerebellar ataxia, polymyositis, Zellweger syndrome, neoplastic cells, and toxic- and drug-induced disorders. Antiepileptic drugs, especially valproate, may impair placental mitochondrial function in pregnant women. (Sarnat HB, Marin-Garcia J. Pathology of mitochondrial encephalomyopathies. Can J Neurol Sci 2005;32:152-166). (Reprints: Harvey B Sarnat MD FRCPC, Alberta Children's Hospital, Pediatric Neurology and Neuropathology, 1820 Richmond Rd SW, Calgary, Alberta T2T 5C7, Canada).

COMMENT. The diagnosis of mitochondrial cytopathy (MC) depends on a classical clinical presentation or phenotype, laboratory findings, and identification of ragged red muscle fibers in tissue biopsy. Mitochondrial DNA may be required for diagnostic confirmation in atypical cases.

\title{
HEPATOCEREBRAL MITOCHONDRIAL DNA DEPLETION
}

Two novel homozygous mutations, G352A and C269T, are documented in the gene for deoxyguanosine kinase (DGK) in 3 children with hepatocerebral mitochondrial DNA depletion syndrome reported from Columbia University College of Physicians and Surgeons, New York; University of Pisa, Italy; University of Toronto, Canada; and University of Melbourne, Australia. All 3 patients developed liver failure and metabolic acidosis in early infancy, one also had cerebral atrophy and nystagmus, a second had microcephaly, hypotonia, and nystagmus, and a third, optic dysplasia with nystagmus and muscle involvement. DGK mutations resulted in truncated polypeptides. In patient 3, who developed multisystem disease, liver transplantation did not prevent brain dysfunction. Systemic involvement portends poor long-term prognosis. (Mancuso M, Ferraris S, Pancrudo J et al. New DGK gene mutations in the hepatocerebral form of mitochondrial DNA depletion syndrome. Arch Neurol May 2005;62:745-747). (Respond: Salvatore DiMauro MD, Room 4-420, Columbia University College of Physicians and Surgeons, $630 \mathrm{~W} 168^{\text {th }} \mathrm{St}$, New York, NY 10032).

COMMENT. Mitochondrial DNA depletion syndrome can affect one, particularly muscle or liver, or multiple organs, and the liver is most frequently affected in DGK gene mutations. Primary mtDNA depletion syndrome is transmitted as an autosomal recessive trait.

\section{THIAMINE-RESPONSIVE CONGENITAL LACTIC ACIDOSIS WITHOUT MC}

Six infants with thiamine-responsive congenital lactic acidosis (CLA), normal pyruvate dehydrogenase complex activity, and no evidence of mitochondrial encephalomyopathy, are reported from Tottori University, Yonago; National Children's Medical Center, Tokyo, and other centers in Japan. Histochemical investigation of muscle from 3 patients showed no ragged red fibers and normal cytochrome $\mathrm{C}$ oxidase activity. Two 